\title{
NOTE SUR LE TISSAGE AUX CARTONS EN CHINE
}

PAR

\section{A. VAN GENNEP.}

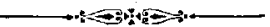

On appelle tissage aux cartons ou aux planchettes (en allemand Brettchenweberei) un procédé de tissage très primitif qui paraît à première vue assez simple, mais qui comporte un grand nombre de variations de détail. En principe, il consiste à faire passer des fils de la même couleur ou de couleurs différentes à travers des cartons ou des planchettes troués, à raison d'un fil par trou. Un peigne mobile tient ces fils séparés de manière que tons les fils qui passent par un même carton passent aussi dans la même rainure du peigne. Le paquet de fils de chaîne est noué au delà du peigne et maintenu écarté à plat en avant des cartons. L'ouvrier passe la navette, portant le fil de trame d'une main, et de l'autre main il tourne les cartons sur leur axe horizontal, perpendiculaire à la chaîne, soit vers lui, soit vers le peigne. Tous les fils tournent ainsi sur enx-mêmes et forment un toron de corde. L'ouvrier donue avec un couteau de bois ou de métal un coup sec sur ce toron, passe de noureau sa navette, tourne les cartons, serre le toron, et ainsi de suite, en donnant régulièrement à sa navette un mouvement de va et vient. La fig. 1 montre l'arrangement des fils à travers les cartons sur un métier à cartons de Tlemcen (département d'Oran) avec tous les matériaux et outils en place. 
Quand les cartons ont été tournés un certain nombre de fois dans un nême sens, les fils sont tellement tordus que la navette ne peut plus passer et que les cartons ne peuvent plus touruer.

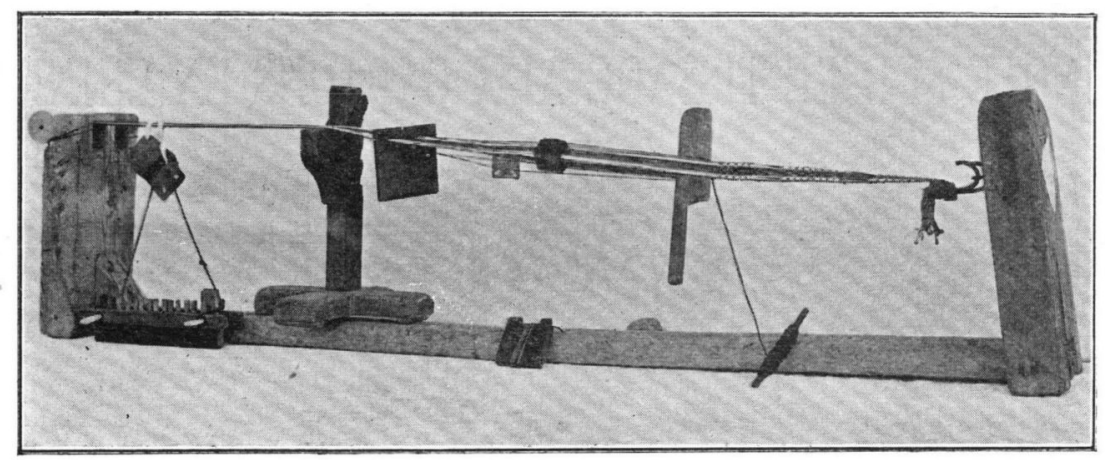

Fig. 1 .

Pour éliminer cet obstacle, il 'y a deux moyens. Ou bien, si les fils de chaine sont assez longs, on peut reculer tous les cartons vers le bout libre. Ou bien, si la chaîne est courte, on peut à partir d'uu certain point tourner les cartons dans l'autre sens. Si l'ourrier les a tournés d'abord, par exemple, de droite à gauche, maintenant il les tournera de gauche à droite. En régularisant ce changement de direction, disons tous les sept passages de navette, on obtient une sorte déterminée de décor à reflets.

Pour plus de détails sur cette technique, je renvoie à un ouvrage bien illustré de $\mathrm{M}^{\text {elle }}$ Lehmann-Filhès, Die Brettchenweberei, $4^{0}$, 56 pages, Berliu, Reimer, 1901, et à mes Études d'Ethnographie Algérienne, $8^{0}$ jésus, Paris, Leroux, 1911, pages 68--82. On trouvera dans ce mémoire (page 80 ) une carte de répartition du tissage aux cartons où la Chine entière se voit légèrement ombrée, attendu que mes renseignements sur la localisation de cette technique dans ce vaste pays étaient nuls. Le plus qu'on savait, c'est qu'il existe au Musée des Postes de Berlin des rubans ou sangles de harnachement de fabrication certainement chinoise et manifestement faits aux 
cartons. Des fragments de ces sangles ont été reproduits par $\mathrm{M}^{\text {clle }}$ Lehmann-Filhès dans son livre page 35 , fig. 48 ; page 39 , fig. 54 ; page 40 , fig. 56 et 57 ; page 43 , fig. 65 . Ils sont tous en laine, sauf celui de la fig. 57 gui est en soie, et sont donnés comme «sangles d'une selle de mandarin sans autre indication de lieu de fabrication ou d'achat.

Avant de dessiner ma carte, je m'étais enquis auprès de personves qui auraient pu se trouver à niême de me fournir des renseiguements, mais saus résultats. Ils me sont venus inopinément. C'est bien par hasard que je suis entré en relations arec M. Volkart, de Saint Gall, qui s'occupe spécialement du tissage aux cartons depuis plusieurs années et qui a bien voulu m'envoyer un échantillon de ruban chinois, ainsi que des photographies, en m'autorisant à les publier; ce dont je le remercie ici vivement. Notre fig. 3 représeute un tisserand aux cartous travaillant à Chang hai, mais origivaire de T'ieu-tsiu.

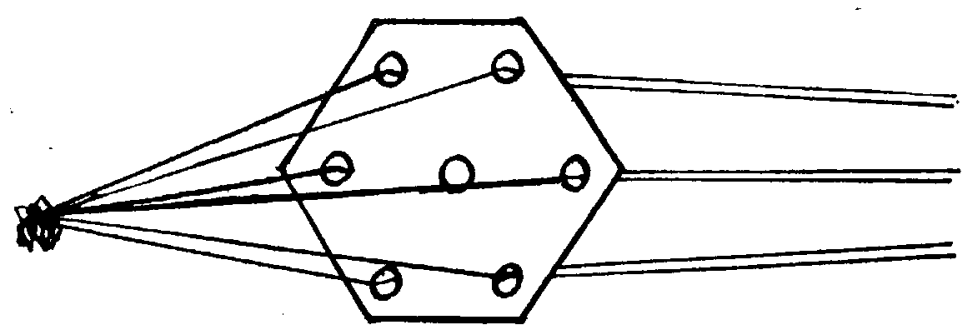

Fig. 2.

Sur cette photographie, on ne roit pas de métier, ce qui correspoud au système actuellement en usage en Finlande et dans quelques régions des pays scandinares ainsi qu'à Tlemcen pour la fabrication des sangles de laine. Les fils sont tous attachés à un objet fixe, branche d'arbre, crochet, etc., d'un côté et de l'autre à la ceinture ou à la jambe de l'ouvrier. A mesure que le tissage avance, l'ouvrier enroule autour de lui le ruban terminé. On remarquera qu'à Chang hai la régularité de tension est obtenue au moyen 
d'une sorte d'échelle servant de support, dont on rapprochera le support figuré à la fig. 1 à gauche et qui était utilisé par un fabricant de sangles en laine de Tlemcen; tout comme l'artisau chinois, l'ourrier tlemcénien travaillait sur une grande longueur de fils.

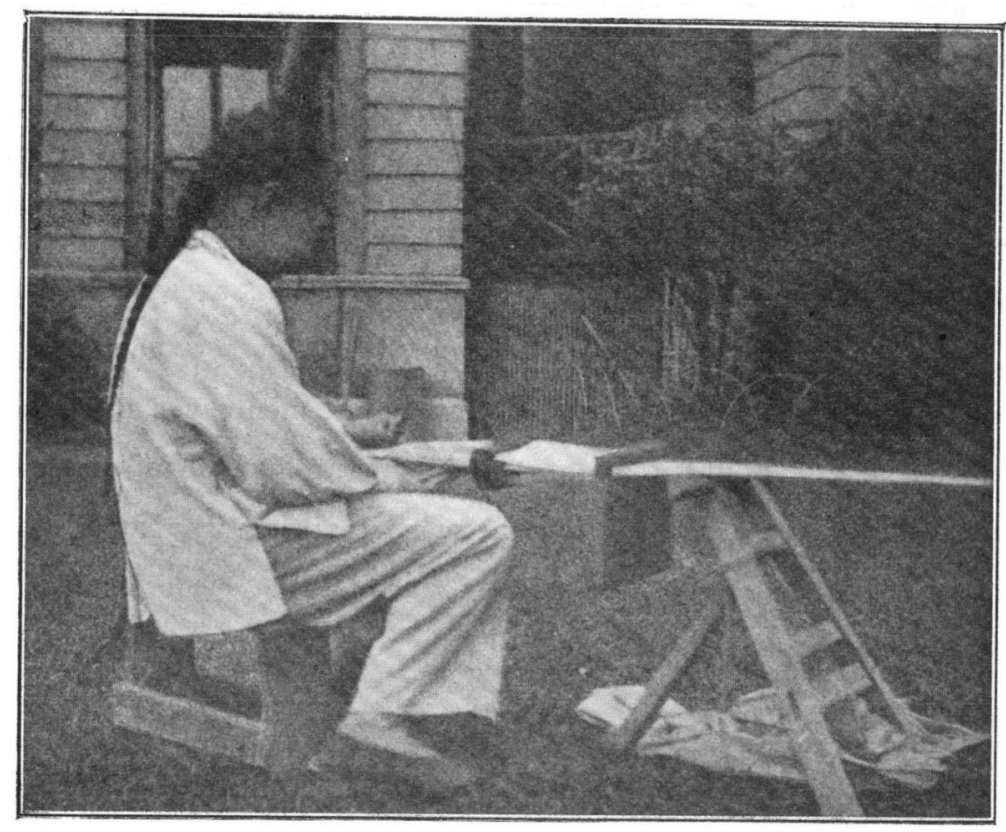

Fig. 3.

Les fils de la trame sont en effet extrêmement longs, à Shanghai, ce qui permet à l'ouvrier de reculer à mesure son peigne et ses cartons sans avoir à recourir au procédé du retournement des cartons dont j'ai parlé tout à l'heure.

En Chine on se sert, non pas de cartons, mais de planchettes de bois. On peut se demander s'il n'a pas existé aussi en Chine des planchettes en ivoire, analogues aux planchettes en os trouvées à Carthage par le père Delattre et qu'on arait prises pour des amulettes.

Alors que partout ailleurs on fait usage de cartons ou de planchettes quadrangulaires, en Chine on utilise des planchettes hexagonales (fig. 2); les deux seuls parallèles que j'aie trourés jusqu'ici 
sont: l'un signalé par $\mathrm{M}^{\text {elle }}$ Lehmann-Filhes pour Mossoul, et l'autre que j'ai constaté cet été à Constantine, chez un ouvrier juif. On ne peut pas voir sur les photographies si les planchettes chinoises sont trouées aussi au centre et si par ce trou central passe un septième fil autour duquel les six fils de coin viennent s'enrouler. L'échantillon acquis à Chang hai que m'a envoyé M. Volkart a été fait à six fils seulement; l'ouvrier de Constantine qui travaille aux cartons hexagonaux m'a afirmé qu'il ne se servait du trou central que pour attacher ensemble ses cartons lorsqu'il a fini de les utiliser, ce que j'ai pu constater par moi-même. Malgré mes instances, cet ourrier, qui avait des commandes pressées, n'a pas voulu me faire un ruban arec ses cartons hexagonaux, de sorte que je ne puis donner de renseignements plus détaillés sur la technique chinoise. Les produits des cartons à six ou à sept trous sont tellement différents au premier abord de ceux des cartons à quatre trous, qu'il faut être averti pour comprend̉re comment ils ont été faits. Les rubans et sangles tissés aux cartons sont à peu près inusables; on peut tailler un trou au centre sans que le reste du ruban se défasse ou se détende.

Le couteau dont se sert l'ourrier chinois est identique à celui en usage dans l'Afrique du Nord et dans l'Asie antérieure; j'ignore sa matière.

A en juger d'après les spécimens découverts et reproduits par $M^{\text {elle }}$ Lehmann-Filhès, les produits du tissage aux planchettes en Chine ne servent que comme pièces de harnachement. En Algérie cet usage est devenu rare, au lieu que la fabrication de rubans de soie (sharit) destinés à servir de ganse intérieure sur les vestes dites "turques» est une petite-industrie relativement importante. Au Caire, en Syrie, au Caucase, la technique sert davantage à faire des ceintures, des tailloles et des jarretières. Peut-être, étant averti, trouvera-t-on qu'en Chine aussi le tissage aux planchettes sert à fabriquer des pièces du costume. 
Il paraît difficile de déterminer la date d'apparition de cette technique en Chine, alors surtout que sa localisation actuelle est encore si peu connue (Chang hai et peut-être T'ien-tsin, puisque l'ouvrier photographié était originaire de cette ville).

Il convient encore de signaler que M. von Le Coq a trouvé le tissage aux cartons dans le Turkestan Oriental. D'après la photographie qu'il a communiquée à $M$. Volkart et que celui-ci m'a envoyée, l'ouvrier travaille sur un métier qui ressemble à celui de Tlemeen par la barre horizontale sur laquelle on met les jambes afin de l'immobiliser, mais qui en diffère en ce que les montants sont simplement des chevilles verticales dans lesquelles s'insèrent d'autres chevilles horizontales; on obtient la tension de la trame en ramenant la pelote de trame près de l'ouvrier. Comme ce métier répond à celui que Breitenstein a rencontré à Bénarès, ainsi qu'au métier en usage à Mossoul, il rentre daus ce que l'on pourrait appeler la catégorie asiatique-antérieure de la technique. Lors de mon deuxième voyage ethnographique en Algérie (1912) j'ai réussi à déterminer l'existence du métier horizontal à montants à Constantine d'une part, à Marakesch, Mogador et Fez de l'autre; M. Joly l'a signalé à Tetouan; cette même technique se rencontre dans la Prusse des premiers siècles de notre ère et dans les pays Scandinaves actuels, mais sans métier horizontal; tout parallèle espagnol et français de cet ordre manque encore; on doit done admettre pour l'Afrique du Nord une importation orientale, ou tout au moins égyptienne ancienne.

Quoi qu'il en soit, l'usage actuel du métier aux cartons à Chang hai et peut-être à T'ien-tsin méritait d'être signalé. Et l'on me permettra de faire appel à la bonne volonté des lecteurs du T'oung pao: peut-être quelqu'un d'entre eux pourra-t-il me signaler d'autres localités en Chine où cette sorte de tissage est exercée comme petite-industrie de sellerie et de passementerie. 